\title{
Global Dynamics of a Cooperative System with Ceiling Density Dependence
}

\author{
Mustafa R. S. Kulenović and Sarah Van Beaver \\ University of Rhode Island \\ Department of Mathematics \\ Kingston, Rhode Island, 02881-0816, USA
}

\begin{abstract}
We investigate the global behavior of the cooperative system

$x_{t+1}=\min \left\{r_{11} x_{t}+r_{12} y_{t}, K_{1}\right\}, y_{t+1}=\min \left\{r_{21} x_{t}+r_{22} y_{t}, K_{2}\right\}, t=0,1, \ldots$

where the initial conditions $x_{0}, y_{0}$ are arbitrary nonnegative numbers. This system models a population comprised of two subpopulations on different patches of land. The model, introduced in [4], considers the minimum between the maximum carrying capacity of each patch $\left(K_{1}\right.$ or $K_{2}$ resp.) and the linear combination of the population from patch $i$ from the last time step with those who migrated to patch $i$ for $i=1,2$. We break the behavior of the system into several cases based on whether the linear combination of the population or maximum carrying capacity is greater. We are able to conclude that either one fixed point will be a global attractor of the interior region of $\mathbb{R}_{+}^{2}$ or there will exist a line of fixed points with the stable manifolds as the basins of attractions. We then extend some of these results to the $n$-dimensional case, first introduced in [2] using similar techniques. We investigate the global behavior of general cooperative system

$$
x_{t+1}^{i}=\min \left\{r_{i 1} x_{t}^{1}+r_{i 2} x_{t}^{2}+\ldots+r_{i i} x_{t}^{i}+\ldots+r_{i n} x_{t}^{n}, K_{i}\right\},
$$

for $i=1,2, \ldots, n$, and $t=0,1, \ldots$ where the initial conditions of $x_{0}^{i}$ are arbitrary nonnegative numbers for $i=1,2, \ldots, n$. We are able to conclude that in some cases one fixed point will be a global attractor of the interior region of $\mathbb{R}_{+}^{n}$.
\end{abstract}

AMS Subject Classifications: 37B25, 37D10,39A10, 39A20, 39A60, 92B05.

Keywords: Attractivity, basin, cooperative, difference equation, invariant manifolds.

Received February 13, 2019; Accepted August 28, 2019

Communicated by Martin Bohner 


\section{Introduction}

We investigate the global behavior of the cooperative system

$$
x_{t+1}=\min \left\{r_{11} x_{t}+r_{12} y_{t}, K_{1}\right\}, \quad y_{t+1}=\min \left\{r_{21} x_{t}+r_{22} y_{t}, K_{2}\right\}, \quad t=0,1, \ldots,
$$

where the initial conditions $x_{0}, y_{0}$ are arbitrary nonnegative numbers. In the original paper [4], Eager et al. define the variables and constants based on a biological model. The model studies a metapopulation consisting of two subpopulations who live on separate patches of land (patch 1 and patch 2), however do not live entirely separate as migration is allowed. The sizes of the subpopulations fluctuate each breeding season due to migration, death, birth, as well as other factors. Additionally, each subpopulation has a maximum known as a carrying capacity as population growth is restricted by the size of the land, food available, etc. In (1.1) the density of the subpopulations is represented by $x_{t}$ (the number of females in patch 1) and $y_{t}$ (the number of females in patch 2) at a time step $t \in \mathbb{N}$. The maximum carrying capacity is represented by $K_{1}, K_{2}>0$ for each patch respectively. The constants of $r_{11} \geq 0, r_{22} \geq 0, r_{12}>0$, and $r_{21}>0$ are the probabilities given as

$$
\begin{aligned}
r_{11}=\left(1-\mu_{1}\right)\left(1+b_{1} f_{1}\right)\left(1-m_{1}\right) & \text { and } \quad r_{22}=\left(1-\mu_{2}\right)\left(1+b_{2} f_{2}\right)\left(1-m_{2}\right), \\
r_{12}=\left(1+b_{2} f_{2}\right) m_{2} \alpha & \text { and } \quad r_{21}=\left(1+b_{1} f_{1}\right) m_{1} \alpha
\end{aligned}
$$

where $b_{1}$ and $b_{2}$ are the probabilities that a female will give birth, $f_{1}$ and $f_{2}$ are the probabilities that the baby is female, $\mu_{1}$ and $\mu_{2}$ are the probabilities of death, $\alpha$ is the probability of a successful migration, and $m_{1}$ and $m_{2}$ are the probabilities of migration for each patch respectively. The linear models in the system are

$$
x_{t+1}=r_{11} x_{t}+r_{12} y_{t}, \quad y_{t+1}=r_{21} x_{t}+r_{22} y_{t},
$$

where $x_{t+1}$ and $y_{t+1}$ represent the current populations at time step $t+1$. Here $r_{11} x_{t}$ is the population of patch 1 that remained from time step $t, r_{12} y_{t}$ is the population that migrated to patch $1, r_{22} y_{t}$ is the population of patch 2 that remained from time step $t$, and $r_{21} x_{t}$ is the population that migrated to patch 2 . Thus, system (1.1) will compute the minimum of the maximum carrying capacity and the linear combination of the females remaining in the population from the last time step with those who migrated to the patch. It is assumed that each subpopulation will increase until it reaches the maximum carrying capacity, also known as the ceiling density dependence.

In this paper we will present the global dynamics of the model for all cases of the parameters. In particular, we obtain the basins of attraction in the two cases of an infinite number of fixed points, which was not covered in [4]. For the four cases of a finite number of fixed points, we will use different, simpler technique of monotone discrete dynamical systems to find the basins of attraction of all fixed points and so to give an alternative proof of the results in [4]. 
The cooperative system (1.1) can be generalized to study a metapopulation consisting of $n$ subpopulations who live on $n$ different patches of land. This system was originally studied in [2]. Such system is as follows

$$
x_{t+1}^{i}=\min \left\{r_{i 1} x_{t}^{1}+r_{i 2} x_{t}^{2}+\ldots+r_{i i} x_{t}^{i}+\ldots+r_{i n} x_{t}^{n}, K_{i}\right\}
$$

for $i=1,2, \ldots, n$ and $t=0,1, \ldots$. The initial conditions $x_{0}^{i}$ are arbitrary nonnegative numbers for $i=1,2, \ldots, n$. For each patch $i$, the density of females in each subpopulation is represented by $x_{t}^{i}$. Additionally, the maximum carrying capacity of each patch will be represented by $K_{i}>0$. In (1.2) the constant $r_{i i} \geq 0$ is the probability of females in patch $i$ surviving from the last time step (and remaining in the patch) as well as the probability of having a female baby born. The constants $r_{i k}>0$ for $k=1,2, \ldots, i-1, i+1, \ldots, n$ are the probabilities of a female migrating to patch $i$ from patch $k$ including the possibility of a newborn baby female migrating during the time step. As with the 2-patch model, we assume that each population will grow linearly until reaching the carrying capacity. We will present the global dynamics of some of the cases using similar techniques to the 2-dimensional system (1.1). We are able to give global results for three cases, in which one fixed point will be a global attractor of the interior region of $\mathbb{R}_{+}^{n}$ using simpler, different techniques than originally used in [2]. The remaining case will be left as conjecture.

Section 2 of this paper contains some basic results on the basins of attraction of the equilibrium solutions of monotone systems and order preserving maps. Section 3 contains the main results giving global dynamics of system (1.1) in all 6 cases, and section 4 contains the main results of the generalized $n$-dimensional system (1.2). It is important to mention that no local or global dynamics of system (1.1) was obtained in [4] in cases 5 and 6 . In fact the global dynamics in these two cases is very interesting and has been observed in number of monotone systems, see $[1,9,10]$.

\section{Some Basic Results for Order Preserving Maps}

In this section we give some basic attractivity results for order preserving maps and systems from $[9,10]$, which will be used in the rest of the paper. See also [5, 6, 12].

We will begin with some definitions and terminology. A first order system of difference equations

$$
\left\{\begin{array}{l}
x_{n+1}=f\left(x_{n}, y_{n}\right) \\
y_{n+1}=g\left(x_{n}, y_{n}\right)
\end{array} \quad, \quad n=0,1, \ldots\right.
$$

where $\mathcal{S} \subset \mathbb{R}^{2}$ has nonempty interior, $(f, g): \mathcal{S} \rightarrow \mathcal{S}, f, g$ are continuous functions is called competitive if $f(x, y)$ is nondecreasing in $x$ and nonincreasing in $y$, and $g(x, y)$ is nonincreasing in $x$ and nondecreasing in $y$. If both $f$ and $g$ are nondecreasing in $x$ and $y$, the system (2.1) is called cooperative. Competitive and cooperative maps are defined similarly. Strongly cooperative systems of difference equations or strongly competitive maps are those for which the functions $f$ and $g$ are coordinate-wise strictly monotone. 
Let $\preceq$ be a partial order on $\mathbb{R}^{n}$ with a nonnegative cone $P$. For $x, y \in \mathbb{R}^{n}$, the ordered interval $\llbracket x, y \rrbracket$ is the set of all $z$ such that $x \preceq z \preceq y$. We say that $x \prec y$ if $x \preceq y$ and $x \neq y$, and $x \ll y$ if $y-x \in$ int $P$, where int $P$ denotes the interior of a set $P$. A map $T$ on a subset of $\mathbb{R}^{n}$ is order preserving if $T(x) \preceq T(y)$ whenever $x \preceq y$, strictly order preserving if $T(x) \prec T(y)$ whenever $x \prec y$, and strongly order preserving if $T(x) \ll T(y)$ whenever $x \prec y$. We say that $\mathcal{B}(x)$ is the basin of attraction of a fixed point $x$ if $T^{n}(y) \rightarrow x$.

Furthermore, we define the south-east partial order as $\preceq_{s e}$ on $\mathbb{R}^{2}$ where $(x, y) \preceq_{\text {se }}$ $(s, t)$ if and only if $x \leq s$ and $y \geq t$. Similarly, we define the north-east partial order as $\preceq_{n e}$ on $\mathbb{R}^{2}$ where $(x, y) \preceq_{n e}(s, t)$ if and only if $x \leq s$ and $y \leq t$.

Let $T: R \rightarrow R$ be a map with a fixed point $\bar{x}$ and let $R^{\prime}$ be an invariant subset of $R$ that contains $\bar{x}$. We say that $\bar{x}$ is stable (asymptotically stable) relative to $R^{\prime}$ if $\bar{x}$ is a stable (asymptotically stable) fixed point of the restriction of $T$ to $R^{\prime}$.

The next result is stated for order-preserving maps on $\mathbb{R}^{n}$ and is given here for completeness. See [5] for a more general version valid in ordered Banach spaces.

Theorem 2.1. For a nonempty set $R \subset \mathbb{R}^{n}$ and $\preceq$ a partial order on $\mathbb{R}^{n}$, let $T: R \rightarrow R$ be an order preserving map, and let $a, b \in R$ be such that $a \prec b$ and $\llbracket a, b \rrbracket \subset R$. If $a \preceq T(a)$ and $T(b) \preceq b$, then $\llbracket a, b \rrbracket$ is invariant interval and

i. There exists a fixed point of $T$ in $\llbracket a, b \rrbracket$.

ii. If $T$ is strongly order preserving, then there exists a fixed point in $\llbracket a, b \rrbracket$ which is stable relative to $\llbracket a, b \rrbracket$.

iii. If there is only one fixed point in $\llbracket a, b \rrbracket$, then it is a global attractor in $\llbracket a, b \rrbracket$ and therefore asymptotically stable relative to $\llbracket a, b \rrbracket$.

We say that $\left\{x_{n}\right\}_{n \in \mathbb{Z}}$ is an entire orbit of a map $T: A \rightarrow A, A \subset \mathbb{R}^{n}$ if $x_{n+1}=$ $T\left(x_{n}\right)$ for all $n \in \mathbb{Z}$. This orbit is said to join $u_{1}$ to $u_{2}$ if $x_{n} \rightarrow u_{1}$ as $n \rightarrow-\infty$ and $x_{n} \rightarrow u_{2}$ as $n \rightarrow \infty$. The following result is for strictly order preserving maps [3,5]. The result is stated for a partial order $\preceq$ in $\mathbb{R}^{n}$, but it also holds in Banach spaces.

Theorem 2.2 (Order Interval Trichotomy of Dancer and Hess, [3]). Let $u_{1} \preceq u_{2}$ be distinct fixed points of a strictly order preserving map $T: A \rightarrow A$, where $A \subset \mathbb{R}^{n}$, and let $I=\llbracket u_{1}, u_{2} \rrbracket \subset A$. Then at least one of the following holds.

(a) Thas a fixed point in I distinct from $u_{1}$ and $u_{2}$.

(b) There exists an entire orbit $\left\{x_{n}\right\}_{n \in \mathbb{Z}}$ of $T$ in $I$ joining $u_{1}$ to $u_{2}$ and satisfying $x_{n} \preceq x_{n+1}$.

(c) There exists an entire orbit $\left\{x_{n}\right\}_{n \in \mathbb{Z}}$ of $T$ in $I$ joining $u_{2}$ to $u_{1}$ and satisfying $x_{n+1} \preceq x_{n}$. 
Corollary 2.3 (See [3]). If $a$ and $b$ are stable fixed points, then there exists a third fixed point in $[a, b]$.

The following result is a direct consequence of Theorem 2.2, see $[9,10]$.

Corollary 2.4. If the nonnegative cone of $\preceq$ is a generalized quadrant in $\mathbb{R}^{n}$, and if $T$ has no fixed points in $\llbracket u_{1}, u_{2} \rrbracket$ other than $u_{1}$ and $u_{2}$, then the interior of $\llbracket u_{1}, u_{2} \rrbracket$ is either a subset of the basin of attraction of $u_{1}$ or a subset of the basin of attraction of $u_{2}$.

The following theorem was proved by Kulenović and Merino [10] for competitive or cooperative systems in the plane, when one of the eigenvalues of the linearized system at an equilibrium (hyperbolic or nonhyperbolic) is by absolute value smaller than 1 while the other has an arbitrary value. This result is useful for determining basins of attraction of fixed points of competitive or cooperative maps.

Theorem 2.5. Let $T$ be a competitive (resp. cooperative) map on a rectangular region $\mathcal{R} \subset \mathbb{R}^{2}$. Let $\overline{\mathrm{x}} \in \mathcal{R}$ be a fixed point of $T$ such that $\Delta:=\mathcal{R} \cap \operatorname{int}\left(\mathcal{Q}_{1}(\bar{x}) \cup \mathcal{Q}_{3}(\overline{\mathrm{x}})\right)$ is nonempty (i.e. " $\overline{\mathrm{x}}$ is not the NW or SE vertex of $\mathcal{R}$ "), and $T$ is strongly competitive (resp. cooperative) on $\Delta$. Suppose that the following statements are true.

a. The map $T$ has a $C^{1}$ extension to a neighborhood of $\overline{\mathrm{x}}$.

b. The Jacobian $J_{T}(\overline{\mathrm{x}})$ of $T$ at $\overline{\mathrm{x}}$ has real eigenvalues $\lambda$, $\mu$ such that $0<|\lambda|<\mu$, where $|\lambda|<1$, and the eigenspace $E^{\lambda}$ associated with $\lambda$ is not a coordinate axis.

Then there exists a curve $\mathcal{C} \subset \mathcal{R}$ through $\overline{\mathrm{x}}$ that is invariant and a subset of the basin of attraction of $\overline{\mathrm{x}}$, such that $\mathcal{C}$ is tangential to the eigenspace $E^{\lambda}$ at $\overline{\mathrm{x}}$, and $\mathcal{C}$ is the graph of a strictly increasing (resp. decreasing) continuous function of the first coordinate on an interval. Any endpoints of $\mathcal{C}$ in the interior of $\mathcal{R}$ are either fixed points or minimal period two points. In the latter case, the set of endpoints of $\mathcal{C}$ is a minimal period two orbit of $T$.

\section{The 2-Patch System}

We will prove the global dynamics of system (1.1) for two patches of land. Let

$$
J=\left[\begin{array}{ll}
r_{11} & r_{12} \\
r_{21} & r_{22}
\end{array}\right]
$$

be the matrix consisting of the constants of system (1.1), and let $\mathcal{T}$ be the cooperative map associated with system (1.1),

$$
\mathcal{T}\left(\left[\begin{array}{l}
x \\
y
\end{array}\right]\right)=\left[\begin{array}{l}
\min \left\{r_{11} x+r_{12} y, K_{1}\right\} \\
\min \left\{r_{21} x+r_{22} y, K_{2}\right\}
\end{array}\right]: \mathbb{R}_{+}^{2} \rightarrow \mathbb{R}_{+}^{2} .
$$

Let $\rho(J)$ denote the spectral radius of $J$ and $\sigma(J)$ denote the set of eigenvalues of $J$. 
Theorem 3.1. The following results hold for system (1.1):

1. Suppose that $\rho(J)<1$ and

$$
K_{1} \geq r_{11} K_{1}+r_{12} K_{2}, \quad K_{2} \geq r_{21} K_{1}+r_{22} K_{2},
$$

where at least one of the inequalities is strict. The fixed point $(0,0)$ is a global attractor.

2. Suppose that $\rho(J)>1,1 \notin \sigma(J)$, and

$$
K_{1} \leq r_{11} K_{1}+r_{12} K_{2}, \quad K_{2} \leq r_{21} K_{1}+r_{22} K_{2},
$$

where at least one of the inequalities is strict. The fixed point $(0,0)$ is unstable while the fixed point $\left(K_{1}, K_{2}\right)$ is a global attractor of the interior region of $\mathbb{R}_{+}^{2}$.

3. Suppose that $\rho(J)>1,1 \notin \sigma(J), r_{22}<1$, and

$$
K_{1} \leq r_{11} K_{1}+r_{12} K_{2}, \quad K_{2}>r_{21} K_{1}+r_{22} K_{2} .
$$

Then the fixed point $(0,0)$ is unstable while the fixed point $\left(K_{1}, K_{1} r_{21} /\left(1-r_{22}\right)\right)$ is a global attractor of the interior region of $\mathbb{R}_{+}^{2}$ where the basin of attraction is $\mathcal{B}\left(K_{1}, K_{1} r_{21} /\left(1-r_{22}\right)\right)$.

4. Suppose that $\rho(J)>1,1 \notin \sigma(J), r_{11}<1$, and

$$
K_{1}>r_{11} K_{1}+r_{12} K_{2}, \quad K_{2} \leq r_{21} K_{1}+r_{22} K_{2} .
$$

Then the fixed point of $(0,0)$ is unstable while the fixed point of $\left(K_{2} r_{12} /(1-\right.$ $\left.\left.r_{11}\right), K_{2}\right)$ is a global attractor of the interior region of $\mathbb{R}_{+}^{2}$ where the basin of attraction is $\mathcal{B}\left(K_{2} r_{12} /\left(1-r_{11}\right), K_{2}\right)$.

5. Suppose that $\rho(J) \geq 1,1 \in \sigma(J), r_{22}<1$, and

$$
K_{1} \leq r_{11} K_{1}+r_{12} K_{2}, \quad K_{2} \geq r_{21} K_{1}+r_{22} K_{2}
$$

System (1.1) has an infinite number of fixed points, $E_{x}=\left\{\left(x, x r_{21} /\left(1-r_{22}\right)\right) \mid 0 \leq\right.$ $\left.x \leq K_{1}\right\}$, each with the stable manifold $\mathcal{W}^{s}\left(E_{x}\right)$ as its basin of attraction.

6. Suppose that $\rho(J) \geq 1,1 \in \sigma(J), r_{11}<1$ and

$$
K_{1} \geq r_{11} K_{1}+r_{12} K_{2}, \quad K_{2} \leq r_{21} K_{1}+r_{22} K_{2} .
$$

System (1.1) has an infinite number of fixed points, $E_{y}=\left\{\left(y r_{12} /\left(1-r_{11}\right), y\right) \mid 0 \leq\right.$ $\left.y \leq K_{2}\right\}$, each with the stable manifold $\mathcal{W}^{s}\left(E_{y}\right)$ as its basin of attraction. 
Proof. 1. Clearly $(0,0)$ is always a fixed point. Using (3.1), the cooperative map $\mathcal{T}$ can be rewritten as

$$
\mathcal{T}\left(\left[\begin{array}{l}
x \\
y
\end{array}\right]\right)=\left[\begin{array}{l}
r_{11} x+r_{12} y \\
r_{21} x+r_{22} y
\end{array}\right] .
$$

Note that no other fixed points will exist as

$$
J\left[\begin{array}{l}
x \\
y
\end{array}\right]=\left[\begin{array}{l}
x \\
y
\end{array}\right]
$$

would have to hold true for some fixed point $(x, y)$. This cannot be the case as $\rho(J)<1$. The map $\mathcal{T}$ has an invariant interval $\llbracket(0,0),\left(K_{1}, K_{2}\right) \rrbracket$. This can be seen as the map is defined on $\mathbb{R}_{+}^{2}$ and the system (1.1) has a maximum carrying capacity $\left(K_{1}, K_{2}\right)$. As $(0,0)$ is the unique fixed point of the invariant interval and $\rho(J)<1$, then

$$
\lim _{t \rightarrow \infty}\left[\begin{array}{l}
x_{t} \\
y_{t}
\end{array}\right]=\left[\begin{array}{l}
0 \\
0
\end{array}\right]
$$

This indeed holds by Theorem 2.1 so $(0,0)$ is a global attractor.

2. The inequalities in (3.1) can be rewritten as

$$
J\left[\begin{array}{l}
K_{1} \\
K_{2}
\end{array}\right] \geq\left[\begin{array}{l}
K_{1} \\
K_{2}
\end{array}\right] .
$$

Therefore as $\left[\begin{array}{l}K_{1} \\ K_{2}\end{array}\right]$ is the minimum, we can conclude that

$$
\mathcal{T}\left(\left[\begin{array}{l}
K_{1} \\
K_{2}
\end{array}\right]\right)=\left[\begin{array}{l}
K_{1} \\
K_{2}
\end{array}\right] .
$$

This results in the fact that $\left(K_{1}, K_{2}\right)$ is a fixed point in addition to $(0,0)$. Using the same reasoning as in the proof of part 1 , another fixed point will only exist if either $x=K_{1}$ and $y \neq K_{2}$ or $x \neq K_{1}$ and $y=K_{2}$. Without loss of generality suppose that $x=K_{1}$ and $y \neq K_{2}$. The map $\mathcal{T}$ gives us that

$$
y=r_{21} x+r_{22} y \quad \Leftrightarrow \quad y=r_{21} K_{1}+r_{22} y \quad \Leftrightarrow \quad y=\frac{r_{21} K_{1}}{1-r_{22}} .
$$

However, this creates a contradiction as the inequality of (3.1) can be rewritten as

$$
K_{2} \leq \frac{r_{21} K_{1}}{1-r_{22}}
$$

Therefore, there are no other fixed points. As $\mathcal{T}\left(\left[\begin{array}{l}x \\ y\end{array}\right]\right) \leq\left[\begin{array}{l}K_{1} \\ K_{2}\end{array}\right]$ for all $(x, y) \in$ $\mathbb{R}_{+}^{2}, \mathcal{T}$ has an invariant interval $\llbracket(0,0),\left(K_{1}, K_{2}\right) \rrbracket$. The fixed point, $(0,0)$ is unstable as $\rho(J)>1$ (see [7]). By Corollary $2.4,\left(K_{1}, K_{2}\right)$ is a global attractor for the interior of $\llbracket(0,0),\left(K_{1}, K_{2}\right) \rrbracket$, that is

$$
\text { int } \llbracket(0,0),\left(K_{1}, K_{2}\right) \rrbracket \subseteq \mathcal{B}\left(\left(K_{1}, K_{2}\right)\right) .
$$


3. Through rearranging one of the inequalities of (3.1) we have

$$
K_{1} \leq r_{11} K_{1}+r_{12} K_{2} \quad \text { and } \quad K_{2}>\frac{r_{21} K_{1}}{1-r_{22}}
$$

We will have the fixed point of $\left(K_{1}, K_{1} r_{21} /\left(1-r_{22}\right)\right)$ when

$$
\mathcal{T}\left(\left[\begin{array}{c}
K_{1} \\
\frac{r_{21} K_{1}}{1-r_{22}}
\end{array}\right]\right)=\left[\begin{array}{c}
K_{1} \\
\frac{r_{21} K_{1}}{1-r_{22}}
\end{array}\right]
$$

holds true. That is if

$$
K_{1} \leq r_{11} K_{1}+r_{12} \frac{r_{21} K_{1}}{1-r_{22}} \quad \text { and } \quad K_{2} \geq r_{21} K_{1}+r_{22} \frac{r_{21} K_{1}}{1-r_{22}}
$$

By the inequalities of (3.7) and the fact that

$$
r_{21} K_{1}+r_{22} \frac{r_{21} K_{1}}{1-r_{22}}=\frac{r_{21} K_{1}}{1-r_{22}}
$$

the second inequality of (3.8) indeed holds. The first inequality of (3.8) can be reduced to

$$
\begin{array}{r}
1 \leq r_{11}+\frac{r_{12} r_{21}}{1-r_{22}} \Leftrightarrow \\
1-r_{22} \leq r_{11}-r_{11} r_{22}+r_{12} r_{21} \\
1 \leq \operatorname{tr}(J)-\operatorname{det}(J) \Leftrightarrow \\
1 \leq \lambda_{1}+\lambda_{2}-\lambda_{1} \lambda_{2} .
\end{array}
$$

Therefore this inequality holds as $\rho(J)>1$ and furthermore, $\left(K_{1}, K_{1} r_{21} /(1-\right.$ $\left.r_{22}\right)$ ) is a fixed point. By using the same argument as in cases 1 and 2 we conclude that there are no other fixed points. By using the fact $\llbracket a, b \rrbracket=\{x: a \leq x \leq b\}$ is an invariant set for $\mathcal{T}$ when $a$ and $b$ are fixed points of a monotone map $\mathcal{T}$, then $\llbracket E_{0}, E_{+} \rrbracket$ is an invariant interval where $E_{0}=(0,0)$ and $E_{+}=\left(K_{1}, K_{1} r_{21} /(1-\right.$ $\left.\left.r_{22}\right)\right)$. As $\rho(J)>1$, then $E_{0}$ is unstable. By Corollary $2.4, E_{+}$is an attractor for the interior of $\llbracket E_{0}, E_{+} \rrbracket$, that is

$$
\text { int } \llbracket E_{0}, E_{+} \rrbracket \subseteq \mathcal{B}\left(E_{+}\right) \text {. }
$$

Since $\left(K_{1}, K_{2}\right)$ is the maximum carrying capacity for the population and $E_{+}$is a fixed point, by using the fact again that $\llbracket a, b \rrbracket$ is an invariant set for monotone map $\mathcal{T}$ when $a$ is fixed point and $b$ is an end point, then $\llbracket E_{+},\left(K_{1}, K_{2}\right) \rrbracket$ is an invariant set. By Theorem 2.1, as $E_{+}$is the only fixed point in the region, then $E_{+}$is an attractor for the interior of $\llbracket E_{+},\left(K_{1}, K_{2}\right) \rrbracket$,

$$
\text { int } \llbracket E_{+},\left(K_{1}, K_{2}\right) \rrbracket \subseteq \mathcal{B}\left(E_{+}\right) .
$$


For all $(x, y) \notin \llbracket E_{0}, E_{+} \rrbracket \cup \llbracket E_{+},\left(K_{1}, K_{2}\right) \rrbracket$ there exists $\left(x_{L}, y_{L}\right) \in \llbracket E_{0}, E_{+} \rrbracket$ and $\left(x_{U}, y_{U}\right) \in \llbracket E_{+},\left(K_{1}, K_{2}\right) \rrbracket$ such that

$$
\left(x_{L}, y_{L}\right) \leq(x, y) \leq\left(x_{U}, y_{U}\right)
$$

Using the fact that $\mathcal{T}$ is monotone,

$$
\mathcal{T}^{n}\left(x_{L}, y_{L}\right) \leq \mathcal{T}^{n}(x, y) \leq \mathcal{T}^{n}\left(x_{U}, y_{U}\right) \text { for } n \in \mathbb{N}
$$

In view of (3.9) and (3.10), $\lim _{n \rightarrow \infty} \mathcal{T}^{n}\left(x_{L}, y_{L}\right)=E_{+}$and $\lim _{n \rightarrow \infty} \mathcal{T}^{n}\left(x_{U}, y_{U}\right)=E_{+}$. Using (3.11) we can conclude that $\lim _{n \rightarrow \infty} T^{n}(x, y)=E_{+}$. Thus, $E_{+}$is a global attractor of the interior of $\llbracket E_{0},\left(K_{1}, K_{2}\right) \rrbracket$.

4. The proof in this case is analogous to the proof of case 3. Through rearranging the inequalities of (3.1) we have

$$
K_{1}>\frac{r_{12} K_{2}}{1-r_{11}} \quad \text { and } \quad K_{2} \leq r_{21} K_{1}+r_{22} K_{2}
$$

We will have the fixed point of $\left(K_{2} r_{12} /\left(1-r_{11}\right), K_{2}\right)$ when

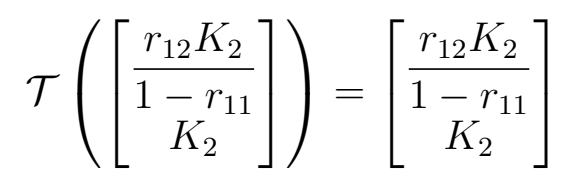

holds true. This will happen if

$$
K_{1} \geq r_{11} \frac{r_{12} K_{2}}{1-r_{11}}+r_{12} K_{2} \quad \text { and } \quad K_{2} \leq r_{21} \frac{r_{12} K_{2}}{1-r_{11}}+r_{22} K_{2}
$$

The first inequality of (3.13) is valid using (3.12) and the fact that

$$
r_{11} \frac{r_{12} K_{2}}{1-r_{11}}+r_{12} K_{2}=\frac{r_{12} K_{2}}{\left(1-r_{11}\right)} .
$$

The second inequality of (3.13) can be reduced to

$$
\begin{array}{r}
1 \leq \frac{r_{21} r_{12}}{1-r_{11}}+r_{22} \Leftrightarrow \\
1-r_{11} \leq r_{21} r_{12}+r_{22}-r_{22} r_{11} \Leftrightarrow \\
1 \leq \operatorname{tr}(J)-\operatorname{det}(J) \Leftrightarrow \\
1 \leq \lambda_{1}+\lambda_{2}-\lambda_{1} \lambda_{2} .
\end{array}
$$

Therefore this inequality holds as $\rho(J)>1$, and furthermore, $\left(K_{2} r_{12} /(1-\right.$ $\left.\left.r_{11}\right), K_{2}\right)$ is a fixed point. By the same arguments as in cases 1 and 2 there are no other fixed points. Furthermore $\llbracket E_{0}, E_{+} \rrbracket$ is an invariant interval where 
$E_{0}=(0,0)^{T}$ and $E_{+}=\left(K_{2} r_{12} /\left(1-r_{11}\right), K_{2}\right)$. As $\rho(J)>1$, then $E_{0}$ is unstable. By Corollary 2.4, $E_{+}$is an attractor for the interior of $\llbracket E_{0}, E_{+} \rrbracket$, that is

$$
\text { int } \llbracket E_{0}, E_{+} \rrbracket \subseteq \mathcal{B}\left(E_{+}\right) \text {. }
$$

As $\left(K_{1}, K_{2}\right)$ is the maximum carrying capacity for the populations and by using the fact that $\llbracket a, b \rrbracket=\{x: a \leq x \leq b\}$ is an invariant set for $\mathcal{T}$ where $a$ is a fixed point and $b$ is the end point of a monotone map $\mathcal{T}$, then $\llbracket E_{+},\left(K_{1}, K_{2}\right) \rrbracket$ is an invariant set. By Theorem 2.1, as $E_{+}$is the only fixed point in $\llbracket E_{+},\left(K_{1}, K_{2}\right) \rrbracket$, then $E_{+}$is an attractor of the interior of $\llbracket E_{+},\left(K_{1}, K_{2}\right) \rrbracket$,

$$
\text { int } \llbracket E_{+},\left(K_{1}, K_{2}\right) \rrbracket \subseteq \mathcal{B}\left(E_{+}\right) .
$$

For all $(x, y) \notin \llbracket E_{0}, E_{+} \rrbracket \cup \llbracket E_{+},\left(K_{1}, K_{2}\right) \rrbracket$ there exists $\left(x_{L}, y_{L}\right) \in \llbracket E_{0}, E_{+} \rrbracket$ and $\left(x_{U}, y_{U}\right) \in \llbracket E_{+},\left(K_{1}, K_{2}\right) \rrbracket$ such that

$$
\left(x_{L}, y_{L}\right) \leq(x, y) \leq\left(x_{U}, y_{U}\right)
$$

By (3.14) and (3.15), we can conclude that $\lim _{n \rightarrow \infty} \mathcal{T}^{n}\left(x_{L}, y_{L}\right)=E^{+}$as well as $\lim _{n \rightarrow \infty} \mathcal{T}^{n}\left(x_{U}, y_{U}\right)=E^{+}$. As $\mathcal{T}$ is monotone, the inequalities of (3.11) from case 3 hold, which implies that $\lim _{n \rightarrow \infty} T^{n}(x, y)=E_{+}$. Thus, $E_{+}$is a global attractor of the interior of $\llbracket E_{0},\left(K_{1}, K_{2}\right) \rrbracket$.

5. If we have that

$$
\mathcal{T}\left(\left[\begin{array}{c}
x \\
\frac{x r_{21}}{\left(1-r_{22}\right)}
\end{array}\right]\right)=\left[\begin{array}{c}
x \\
x r_{21} \\
\left(1-r_{22}\right)
\end{array}\right] \text { for } 0 \leq x \leq K_{1},
$$

then $\left\{\left(x, x r_{21} /\left(1-r_{22}\right)\right) \mid 0 \leq x \leq K_{1}\right\}$ are fixed points. As a reminder, in case 3 we proved that $\left(K_{1}, K_{1} r_{21} /\left(1-r_{22}\right)\right)$ is a fixed point. There will exist an infinite number of fixed points whenever $x \neq K_{1}$ and

$$
x=r_{11} x+\frac{r_{12} r_{21} x}{\left(1-r_{22}\right)} \quad \text { and } \quad \frac{x r_{21}}{\left(1-r_{22}\right)}=r_{21} x+r_{22} \frac{x r_{21}}{\left(1-r_{22}\right)} .
$$

The second equality of (3.16) clearly holds true. The first equality of (3.16) can be rewritten as

$$
\begin{array}{r}
1=r_{11}+\frac{r_{12} r_{21}}{\left(1-r_{22}\right)} \Leftrightarrow \\
1-r_{22}=r_{21} r_{12}+r_{11}-r_{22} r_{11} \Leftrightarrow \\
1 \leq \operatorname{tr}(J)-\operatorname{det}(J)
\end{array}
$$

By rearranging the inequalities from (3.1) we have that

$$
K_{1} \leq \frac{r_{12}}{1-r_{11}} K_{2}
$$


Since $K_{1}$ and $K_{2}$ are nonnegative and $r_{12}>0$, then $r_{11}<1$. The fact that $r_{11}, r_{22}<1$ implies that $0<\operatorname{tr}(J)<2$. As $\operatorname{tr}(J)=\lambda_{1}+\lambda_{2}$, then $0<\lambda_{1}+\lambda_{2}<2$ as well. We can conclude that as $\lambda_{1}=1$ (by $1 \in \sigma(J)$ ), then $\lambda_{2}<1$, and moreover, $\rho(J)=1$. Therefore we can conclude that $1 \leq \operatorname{tr}(J)-\operatorname{det}(J)$ as $\rho(J)=1$ and $1 \in \sigma(J)$, and furthermore, $\left\{\left(x, x r_{21} /\left(1-r_{22}\right)\right) \mid 0 \leq x \leq K_{1}\right\}$ are fixed points.

Next as $\mathcal{T}$ is defined on $\mathbb{R}_{+}^{2}$ and has a maximum of $\left(K_{1}, K_{2}\right)$, then $\mathcal{T}$ is defined on the rectangular region $\mathcal{R}=\llbracket(0,0),\left(K_{1}, K_{2}\right) \rrbracket \subset \mathbb{R}^{2}$. Let $\bar{x}$ be a fixed point such that

$$
\bar{x} \in\left\{\left(x, x \cdot r_{21} /\left(1-r_{22}\right)\right) \mid 0 \leq x \leq K_{1}\right\} .
$$

Then $\bar{x}$ will be in $\Delta:=\mathcal{R} \cap \operatorname{int}\left(\mathcal{Q}_{1}(\bar{x}) \cap \mathcal{Q}_{3}(\bar{x})\right)$ as all of the fixed points are in $\mathcal{R}$ and in the first quadrant of $\mathbb{R}^{2}$. As (1.1) is linear in $\mathcal{R}$, then the map is strictly monotone on $\mathcal{R}$, and moreover strongly cooperative. The map $\mathcal{T}$ has a $C^{1}$ extension to a neighborhood of $\bar{x}$ as the map is linear. Since $\lambda_{1}=1$ and $\lambda_{2}<1$, by Theorem 2.5, there exists a curve $\mathcal{C} \subset \mathcal{R}$ through $\bar{x}$ that is invariant and a subset of the basin of attraction of $\bar{x}$ such that $\mathcal{C}$ is the graph of a strictly decreasing continuous function of the first coordinate on an interval, and any endpoints of $\mathcal{C}$ in the interior of $\mathcal{R}$ are either fixed points or minimal period two points. This holds for every fixed point in $\left\{\left(x, x r_{21} /\left(1-r_{22}\right)\right) \mid 0 \leq x \leq K_{1}\right\}$ as $\bar{x}$ was arbitrary. Thus, $\left\{\left(x, x r_{21} /\left(1-r_{22}\right)\right) \mid 0 \leq x \leq K_{1}\right\}$ will have a stable manifold. By using [1, Theorem 3.4], we can prove that the fixed point depends continuously on the initial point.

6. We want to prove that the fixed points $\left\{\left(y r_{12} /\left(1-r_{11}\right), y\right) \mid 0 \leq y \leq K_{2}\right\}$ will have the stable manifold. This proof will be analogous to the proof of case 5. If we have that

$$
\mathcal{T}\left(\left[\begin{array}{c}
y r_{12} \\
\left(1-r_{11}\right) \\
y
\end{array}\right]\right)=\left[\begin{array}{c}
\frac{y r_{12}}{\left(1-r_{11}\right)} \\
y
\end{array}\right] \text { for } 0 \leq y \leq K_{2}
$$

then $\left\{\left(y r_{12} /\left(1-r_{11}\right), y\right) \mid 0 \leq y \leq K_{2}\right\}$ are fixed points. In case 4 we proved that $\left(K_{2} r_{12} /\left(1-r_{11}\right), K_{2}\right)$ is a fixed point. When $y \neq K_{2}$ there will be an infinite number of fixed points if

$$
\frac{y r_{12}}{\left(1-r_{11}\right)}=\frac{y r_{11} r_{12}}{\left(1-r_{11}\right)}+r_{12} y \quad \text { and } \quad y=\frac{y r_{12} r_{21}}{\left(1-r_{11}\right)}+r_{22} y
$$

Note that the first equality of (3.17) clearly holds true. The second equality of (3.17) can be rewritten as

$$
\begin{array}{r}
1=\frac{r_{12} r_{21}}{\left(1-r_{11}\right)}+r_{22} \Leftrightarrow \\
1-r_{11}=r_{21} r_{12}+r_{22}-r_{22} r_{11} \Leftrightarrow
\end{array}
$$




$$
1 \leq \operatorname{tr}(J)-\operatorname{det}(J)
$$

By rearranging one of the inequalities from (3.1) we get

$$
K_{2} \leq \frac{r_{21}}{1-r_{22}} K_{1}
$$

Since $K_{1}$ and $K_{2}$ are nonnegative and $r_{21}>0$, then $r_{22}<1$. The fact that $r_{11}, r_{22}<1$ implies that $0<\operatorname{tr}(J)<2$. As $\operatorname{tr}(J)=\lambda_{1}+\lambda_{2}$, then $0<\lambda_{1}+\lambda_{2}<2$ as well. We can conclude that as $\lambda_{1}=1$ (by $1 \in \sigma(J)$ ), $\lambda_{2}<1$, and moreover, $\rho(J)=1$. Therefore $1 \leq \operatorname{tr}(J)-\operatorname{det}(J)$ holds as $\rho(J)=1$ and $1 \in \sigma(J)$, and furthermore, $\left\{\left(y r_{12} /\left(1-r_{11}\right), y\right) \mid 0 \leq y \leq K_{2}\right\}$ are fixed points. The remainder of the proof is analogous to case 5 , and thus completes the proof.

\section{The $n$-Patch System}

We will prove the global dynamics in some cases of system (1.2) for $n$ patches of land. Let $M$ be the matrix comprised of constants of (1.2):

$$
M=\left[\begin{array}{cccc}
r_{11} & r_{12} & \ldots & r_{1 n} \\
r_{21} & r_{22} & \ldots & r_{2 n} \\
\vdots & \vdots & \ddots & \vdots \\
r_{n 1} & r_{n 2} & \ldots & r_{n n}
\end{array}\right]
$$

Define $\mathcal{T}$ to be the cooperative map associated with system (1.2)

$$
\mathcal{T}\left(\left[\begin{array}{c}
x^{1} \\
\vdots \\
x^{n}
\end{array}\right]\right)=\left[\begin{array}{c}
\min \left\{r_{11} x^{1}+r_{12} x^{2}+\ldots+\ldots+r_{1 n} x^{n}, K_{1}\right\} \\
\vdots \\
\min \left\{r_{n 1} x^{1}+r_{n 2} x^{2}+\ldots+\ldots+r_{n n} x^{n}, K_{n}\right\}
\end{array}\right]: \mathbb{R}_{+}^{n} \rightarrow \mathbb{R}_{+}^{n} .
$$

Additionally, let $\bar{x}$ be an equilibrium point of the system (1.2). By [2, Lemmas 3.2, 3.3, and 3.4] we have that

Lemma 4.1. 1. If $r_{i 1} K_{1}+r_{i 2} K_{2}+\ldots+r_{i i} K_{i}+\ldots+r_{i n} K_{n} \leq K_{i}$ for each $i=$ $1,2, \ldots, n$ with at least one strict inequality, then $\rho(M)<1$.

2. If $r_{i 1} K_{1}+r_{i 2} K_{2}+\ldots+r_{i i} K_{i}+\ldots+r_{i n} K_{n} \geq K_{i}$ for each $i=1,2, \ldots$, n with at least one strict inequality, then $\rho(M)>1$.

We state the results of [2, Theorem 3.5].

Lemma 4.2. 1. If $\rho(M)<1$, then $(0, \ldots, 0)$ is the only fixed point. 
2. If $\rho(M)>1$ and $r_{i 1} K_{1}+r_{i 2} K_{2}+\ldots+r_{i i} K_{i}+\ldots+r_{i n} K_{n} \geq K_{i}$ for each $i=1,2, \ldots, n$ with at least one strict inequality, then the only fixed points are $(0, \ldots, 0)$ and $\left(K_{1}, \ldots, K_{n}\right)$.

3. If $\rho(M)>1$ and both $r_{i 1} K_{1}+r_{i 2} K_{2}+\ldots+r_{i i} K_{i}+\ldots+r_{i n} K_{n} \leq K_{i}$ and $r_{j 1} K_{1}+r_{j 2} K_{2}+\ldots+r_{j j} K_{j}+\ldots+r_{j n} K_{j} \geq K_{j}$ for $i, j$ in $1,2, \ldots, n$ with some of the inequalities strict, then the only fixed points are $(0, \ldots, 0)$ and there exist one nonzero fixed point, $E_{+}$, with some patches at capacity and some below.

We can now formulate a theorem about the global stability in some cases and provide new proofs based on the theory of monotone maps.

Theorem 4.3. The following result holds for system (1.2):

1. Suppose that

$$
r_{i 1} K_{1}+r_{i 2} K_{2}+\ldots+r_{i i} K_{i}+\ldots+r_{i n} K_{n} \leq K_{i}
$$

for each $i=1,2, \ldots, n$ with at least one strict inequality. Then $\rho(M)<1$ and the fixed point $(0, \ldots, 0)$ is a global attractor.

2. Suppose that

$$
r_{i 1} K_{1}+r_{i 2} K_{2}+\ldots+r_{i i} K_{i}+\ldots+r_{i n} K_{n} \geq K_{i}
$$

for each $i=1,2, \ldots, n$ with at least one strict inequality. Then $\rho(M)>1$ and the fixed point $(0, \ldots, 0)$ is unstable, while the fixed point $\left(K_{1}, \ldots, K_{n}\right)$ is a global attractor of the interior region of $\mathbb{R}_{+}^{n}$.

3. Suppose that $\rho(J)>1$ and $1 \notin \sigma(J)$ and

$$
\begin{array}{r}
r_{i 1} K_{1}+r_{i 2} K_{2}+\ldots+r_{i i} K_{i}+\ldots+r_{i n} K_{n} \leq K_{i} \\
r_{j 1} K_{1}+r_{j 2} K_{2}+\ldots+r_{j j} K_{j}+\ldots+r_{j n} K_{j} \geq K_{j}
\end{array}
$$

for $i$ and $j$ in $1,2, \ldots, n$ with some of the inequalities strict. Then the fixed point $(0, \ldots, 0)$ is unstable while the nonzero fixed point, $E_{+}$is a global attractor of the interior region of $\mathbb{R}_{+}^{n}$.

Proof. 1. The point $(0, \ldots, 0)$ will always be a fixed point of (1.2). We can rewrite the inequalities of (4.1) as

$$
\left[\begin{array}{c}
K_{1} \\
\vdots \\
K_{n}
\end{array}\right] \leq M\left[\begin{array}{c}
K_{1} \\
\vdots \\
K_{n}
\end{array}\right]
$$


We can rewrite the cooperative map as

$$
\mathcal{T}\left(\left[\begin{array}{c}
x^{1} \\
\vdots \\
x^{n}
\end{array}\right]\right)=\left[\begin{array}{c}
r_{11} x^{1}+r_{12} x^{2}+\ldots+\ldots+r_{1 n} x^{n} \\
\vdots \\
r_{n 1} x^{1}+r_{n 2} x^{2}+\ldots+\ldots+r_{n n} x^{n}
\end{array}\right]
$$

The map $\mathcal{T}$ will have an invariant interval $\llbracket(0, \ldots, 0),\left(K_{1}, \ldots, K_{n}\right) \rrbracket$ as the map is defined on the region of $\mathbb{R}_{+}^{n}$ and has a maximum carrying capacity $\left(K_{1}, \ldots, K_{n}\right)$. As $(0, \ldots, 0)$ is the unique fixed point of the invariant interval and $\rho(M)<1$, then

$$
\lim _{t \rightarrow \infty}\left[\begin{array}{c}
x_{t}^{1} \\
\vdots \\
x_{t}^{n}
\end{array}\right]=\left[\begin{array}{c}
0 \\
\vdots \\
0
\end{array}\right] .
$$

Indeed by Theorem $2.1,(0, \ldots, 0)$ is a global attractor.

2. As a result of the inequalities of (4.2),

$$
M\left[\begin{array}{c}
K_{1} \\
\vdots \\
K_{n}
\end{array}\right] \geq\left[\begin{array}{c}
K_{1} \\
\vdots \\
K_{n}
\end{array}\right]
$$

Thus in addition to $(0, \ldots, 0),\left(K_{1}, \ldots, K_{n}\right)$ is a fixed point of (1.2) under the conditions of (4.2) as we can conclude

$$
\mathcal{T}\left(\left[\begin{array}{c}
K_{1} \\
\vdots \\
K_{n}
\end{array}\right]\right)=\left[\begin{array}{c}
K_{1} \\
\vdots \\
K_{n}
\end{array}\right] .
$$

The map $\mathcal{T}$ has an invariant interval $\llbracket(0, \ldots, 0),\left(K_{1}, \ldots, K_{n}\right) \rrbracket$ as the map is defined on $\mathcal{R}_{+}^{n}$ and for all $\left(x_{1}, \ldots, x_{n}\right) \in \mathbb{R}_{+}^{n}, \mathcal{T}\left(\left[\begin{array}{c}x_{1} \\ \vdots \\ x_{n}\end{array}\right]\right) \leq\left[\begin{array}{c}K_{1} \\ \vdots \\ K_{n}\end{array}\right]$. The fixed point $(0, \ldots, 0)$ is unstable as $\rho(M)>1$ (see [7]). By Corollary 2.4, $\left(K_{1}, \ldots, K_{n}\right)$ is the global attractor for the interior of $\llbracket(0, \ldots, 0),\left(K_{1}, \ldots, K_{n}\right) \rrbracket$, that is

$$
\text { int } \llbracket(0, \ldots, 0),\left(K_{1}, \ldots, K_{n}\right) \rrbracket \subseteq \mathcal{B}\left(\left(K_{1}, \ldots, K_{n}\right)\right) .
$$

3. In addition to the fixed point $E_{0}=(0, \ldots, 0)$ there exists one more fixed point $E_{+}$ by Lemma 4.2. As $\mathcal{T}$ is a monotonic map and both $E_{0}$ and $E_{+}$are fixed points, then $\llbracket E_{0}, E_{+} \rrbracket$ is an invariant interval. The fixed point $E_{0}$ is unstable as $\rho(M)>1$. By Corollary 2.4, $E_{+}$is an attractor for the interior region of $\llbracket E_{0}, E_{+} \rrbracket$, that is

$$
\text { int } \llbracket E_{0}, E_{+} \rrbracket \subseteq \mathcal{B}\left(E_{+}\right) \text {. }
$$


Again as $\mathcal{T}$ is a monotonic map and additionally as $E_{+}$is a fixed point while $\left(K_{1}, \ldots, K_{n}\right)$ is the maximum carrying capacity, $\llbracket E_{+},\left(K_{1}, \ldots, K_{n}\right) \rrbracket$ is an invariant interval. By Theorem 2.1, since $E_{+}$is the only fixed point in the interval, then $E_{+}$is an attractor for the interior of $\llbracket E_{+},\left(K_{1}, \ldots, K_{n}\right) \rrbracket$, that is

$$
\text { int } \llbracket E_{+},\left(K_{1}, \ldots, K_{n}\right) \rrbracket \subseteq \mathcal{B}\left(E_{+}\right) .
$$

It remains to prove that $E_{+}$is the attractor for the interior of the invariant interval $\llbracket E_{0},\left(K_{1}, \ldots, K_{n}\right) \rrbracket$. From above we know that for all $\left(x_{1}, \ldots, x_{n}\right) \notin \llbracket E_{0}, E_{+} \rrbracket \cup$ $\llbracket E_{+},\left(K_{1}, \ldots, K_{n}\right) \rrbracket$ there exists $\left(x_{1}^{L}, \ldots, x_{n}^{L}\right) \in \llbracket E_{0}, E_{+} \rrbracket$ and $\left(x_{1}^{U}, \ldots, x_{n}^{U}\right) \in$ $\llbracket E_{+},\left(K_{1}, \ldots, K_{n}\right) \rrbracket$ such that

$$
\left(x_{1}^{L}, \ldots, x_{n}^{L}\right) \leq\left(x_{1}, \ldots, x_{n}\right) \leq\left(x_{1}^{U}, \ldots, x_{n}^{U}\right) .
$$

As $\mathcal{T}$ is monotone,

$$
\mathcal{T}^{k}\left(\left(x_{1}^{L}, \ldots, x_{n}^{L}\right)\right) \leq \mathcal{T}^{k}\left(\left(x_{1}, \ldots, x_{n}\right)\right) \leq \mathcal{T}^{k}\left(\left(x_{1}^{U}, \ldots, x_{n}^{U}\right)\right) \text { for } k \in \mathbb{N} .
$$

As both (4.3) and (4.4) hold, we can conclude that $\lim _{k \rightarrow \infty} \mathcal{T}^{k}\left(\left(x_{1}^{L}, \ldots, x_{n}^{L}\right)\right)=E_{+}$ and $\lim _{k \rightarrow \infty} \mathcal{T}^{k}\left(\left(x_{1}^{U}, \ldots, x_{n}^{U}\right)\right)=E_{+}$. Using the inequalities (4.5) we conclude that $\lim _{k \rightarrow \infty} \mathcal{T}^{k}\left(x_{1}, \ldots, x_{n}\right)=E_{+}$. Therefore $E_{+}$is a global attractor of the interior of $\llbracket E_{0},\left(K_{1}, \ldots, K_{n}\right) \rrbracket$ and this concludes the proof.

As at this point in time the stable manifold theory does not extend to $\mathbb{R}_{+}^{n}, n>2$, we leave cases 5 and 6 from the 2-dimensional case as conjectures.

Conjecture 4.4. If $\rho(M) \geq 1,1 \in \sigma(M)$, and

$$
\begin{aligned}
& r_{i 1} K_{1}+r_{i 2} K_{2}+\ldots+r_{i i} K_{i}+\ldots+r_{i n} K_{n} \leq K_{i} \\
& r_{j 1} K_{1}+r_{j 2} K_{2}+\ldots+r_{j j} K_{j}+\ldots+r_{j n} K_{j} \geq K_{j}
\end{aligned}
$$

for $i, j \in\{1,2, \ldots, n\}$ with no strict inequalities, then there will be infinite fixed points with the corresponding stable manifold as its basins of attraction.

\section{References}

[1] Dž. Burgić, S. Kalabušić, and M. R. S. Kulenović, Non-hyperbolic dynamics for competitive systems in the plane and global period-doubling bifurcations, $A d v$. Dyn. Syst. Appl., 3(2008), 229-249.

[2] J. Callahan, R. Rebarber, E. Strawbridge, B. Tenhumberg, and S. Yuan, Analysis of a coupled n-patch population model with ceiling density dependence. Inter. J. Difference Equ. 10 (2015), 137-159. 
[3] E. Dancer and P. Hess, Stability of fixed points for order preserving discrete-time dynamical systems, J. Reine Angew Math.,(1991), 1991: 419, 125-139.

[4] E. Eager, M. Hebert, E. Hellwig, F. Hernandez, R. Rebarber, B. Tenhumberg, and B. Wigianto, Global Attracting Equilibria for Coupled Systems with Ceiling Density Dependence, Inter. J. Difference Equ. 8(2013), 179-193.

[5] P. Hess, Periodic-parabolic boundary value problems and positivity, Pitman Research Notes in Mathematics Series, 247. Longman Scientific and Technical, Harlow; copublished in the United States with John Wiley and Sons, Inc., New York, 1991.

[6] M. Hirsch and H. L. Smith, Monotone Maps: A Review, J. Difference Equ. Appl. 11(2005), 379-398.

[7] M. R. S. Kulenović and O. Merino, Discrete Dynamical Systems and Difference Equations with Mathematica, Chapman \& Hall/CRC, Boca Raton, London, 2002.

[8] M. R. S. Kulenović and O. Merino, Competitive-Exclusion versus CompetitiveCoexistence for Systems in the Plane, Discrete Cont. Dyn. Syst.Ser. B 6(2006), 1141-1156.

[9] M. R. S. Kulenović and O. Merino, Global Bifurcation for Competitive Systems in the Plane, Discrete Contin. Dyn. Syst. B, 12 (2009), 133-149.

[10] M. R. S. Kulenović and O. Merino, Invariant manifolds for competitive discrete systems in the plane. Internat. J. Bifur. Chaos Appl. Sci. Engrg. 20 (2010), no. 8, 2471-2486.

[11] V. Lakshmikantham and D. Trigiante, Theory of difference equations: numerical methods and applications. Second edition. Monographs and Textbooks in Pure and Applied Mathematics, 251. Marcel Dekker, Inc., New York, 2002.

[12] H. L. Smith, Planar Competitive and Cooperative Difference Equations, J. Difference Equ. Appl. 3(1998), 335-357. 